\title{
RELATIONSHIP OF DIABETIC MICROVASCULAR COMPLICATIONS TO OUTCOME IN PANRETINAL PHOTOCOAGULATION TREATMENT OF PROLIFERATIVE DIABETIC RETINOPATHY
}

\author{
M. F. CORDEIRO, M. R. STANFORD, P. M. PHILLIPS and J. S. SHILLING \\ London
}

\begin{abstract}
SUMMARY
Argon laser panretinal photocoagulation for proliferative diabetic retinopathy was shown in the Diabetic Retinopathy Study and Early Treatment Diabetic Retinopathy Study to reduce the incidence of blindness by $50 \%$ with relatively small amounts of treatment. However, some diabetics require much more extensive photocoagulation for control of proliferative disease. We attempted to determine risk factors for poor response to treatment with panretinal photocoagulation (PRP) by studying outcome in relation to the argon laser burn count and the presence of diabetic vascular complications. Sixty-six consecutively treated eyes undergoing PRP were studied, of which $57 \%$ showed resolution of new vessels 6 weeks after treatment. This was significantly related to the total amount of laser treatment given (mean no. in regressed eyes 5800 burns, non-regressed 3510 burns; $p<0.05)$. Renal disease and age $(<50$ years) were identified as risk factors for non-regression $(p<0.05)$; hypertension, neuropathy, duration of disease and insulin dependence had no significant effect on outcome. We conclude that regression of proliferative disease is significantly related to the cumulative total number of laser burns applied and that successful laser photocoagulation in patients with diabetic renal disease requires considerably more treatment than that suggested by earlier studies.
\end{abstract}

Proliferative diabetic retinopathy (PDR) is a microvascular complication of diabetes mellitus characterised by the formation of new blood vessels on the retina, optic disc or iris with a propensity to rupture, bleed and fibrose. Although renal and arterial disease are the main causes of death in diabetics, the ocular complications of the disease are a major

Correspondence to: Mr J. S. Shilling, Department of Ophthalmology, Greenwich District Hospital, Vanbrugh Hill, London SE18, UK. determinant of disability. Diabetics are about $10-20$ times more likely to go blind than non-diabetics and this disease accounts for about $7 \%$ of the newly registered blind in the UK. ${ }^{1}$ Diabetes is now the commonest single cause of blindness in patients under 65 years in Great Britain ${ }^{2}$ and the British Diabetic Association estimate the annual percentage of newly registerable blindness caused by diabetes to be $11.9 \%$ (personal communication). The main cause of blindness in diabetic patients is retinal disease resulting from severe maculopathy, recurrent vitreous haemorrhages and tractional retinal detachments.

Before the development of laser photocoagulation, the visual prognosis of patients with PDR was very poor. In the 1960s, two separate studies showed the incidence of blindness amongst patients with PDR to be at least $50 \%$, within 5 years (on average 3.2 years) of onset of the disease. ${ }^{1,3}$ The introduction of laser photocoagulation revolutionised the management of PDR and its effectiveness was clearly demonstrated by the first large clinical trial of laser photocoagulation for diabetic retinopathy, the Diabetic Retinopathy Study (DRS). ${ }^{4,5}$ After 6 years of follow-up, a $50 \%$ treatment effect was demonstrated: the untreated eyes had a $34.2 \%$ cumulative rate of severe visual loss $(\mathrm{VA}<5 / 200)$, whereas the cumulative rate in the eyes treated with argon laser (800-1600 burns of $500 \mu \mathrm{m}$ spot size or $500-1000$ burns of $1000 \mu \mathrm{m}$ spot size, 0.1 seconds at one sitting) was $17.5 \%$. A $50 \%$ treatment effect was also observed in the Early Treatment Diabetic Retinopathy Study (ETDRS), ${ }^{6,7}$ which used the same argon laser parameters as the DRS for 'full' panretinal photocoagulation (PRP). The findings of the DRS and ETDRS created a standard of management for all eyes with high risk factors for PDR. They suggested that the number of burns applied in 
their 'full' laser PRP protocol was sufficient to induce regression of PDR, and therefore represented an adequate treatment regimen.

Subsequent laser photocoagulation studies have shown that some patients with PDR require much more extensive PRP to induce neovascular regression. ${ }^{8-10}$ However, no trial has investigated patient response in relation to either the total number of laser burns applied or systemic disease. In an attempt to identify risk factors that might predict an unfavourable outcome of PRP, we investigated firstly, whether the response of PDR was affected by the cumulative total of argon laser PRP, and secondly, whether the presence of systemic diabetic vascular complications influenced treatment response.

\section{METHODS}

A prospective study was performed on consecutive patients with PDR requiring laser treatment attending the Medical Retinal Clinic at Greenwich District Hospital over a 6-month period.

All patients had fundal examination through dilated pupils, performed by at least two of the authors (J.S.S., M.R.S.) to validate the diagnosis. Proliferative disease was defined for the purposes of the study as active disc neovascularisation; patients with new vessels elsewhere only were excluded. Both newly diagnosed patients and patients with a history of treated PDR were included. In newly diagnosed patients all new disc vessels were defined as 'active', and in previously treated patients activity was identified by the re-appearance of new vessels.

Each patient was formally assessed using the parameters shown in Table I by careful review of patient's clinical notes and direct enquiry about the presence of diabetic complications, with special attention to vascular diseases. Patients were considered as suffering from hypertension only if they were on anti-hypertensive therapy. Diabetic renal disease was identified by documented evidence of severe renal impairment as determined by frank proteinuria or renal failure requiring dialysis. Patients were asked about neurological symptoms of paraesthesia, nocturnal cramps and autonomic dysfunction to determine the presence of symptomatic diabetic neuropathy. In cases where it was unclear whether these conditions existed, the patients' diabetic notes were reviewed. All patients in this study were under review by the diabetic clinic of the hospital.

Treatment in the index session with argon laser photocoagulation was performed; newly diagnosed patients were given approximately $1500-2000$ burns per eye using a 400-500 $\mu \mathrm{m}$ spot size (at laser control), 0.1 second duration, and with a power setting sufficient to achieve retinal pigment epithelial blanching. Patients with media opacity that did not allow adequate visualisation of laser burns were specifically excluded from the study. A Rodenstock lens was used and treatment was given so that burns were approximately one half-burn apart. In previously treated patients, laser treatment was applied to areas of untreated retina to fill in the gaps with confluent treatment (i.e. overlapping of circular burns), and where no gaps existed, as in cases of severe, refractory disease, PRP was applied over old laser scars.

For each patient, the cumulative number of laser burns received was calculated from the sum of all previously applied burns added to the total number given in the index laser session. Patients were reviewed, independent of the laser operator, in clinic 6 weeks after PRP, when fundal examination was again performed to assess the response to treatment. Success was defined as evidence of disc new vessel regression, as evidenced by a decrease in vessel calibre and presence of less bulbar tips accompanied by increasing fibrosis. ${ }^{11}$

Statistical analysis was performed using logistic regression. This was done defining disc neovascular regression as the dependent variable with likelihood odds ratios calculated for all other parameters analysed using backward stepwise analysis.

Table I. Distribution of patient characteristics

\begin{tabular}{|c|c|c|c|}
\hline Characteristic & Incidence (\%) & Mean & Range \\
\hline $\begin{array}{l}\text { Male } \\
\text { IDDM }\end{array}$ & $\begin{array}{l}43(65.2) \\
33(50)\end{array}$ & & \\
\hline $\begin{array}{l}\text { Duration of diabetes (years) } \\
(>10 \text { years })\end{array}$ & $35(53)$ & 13.3 & $1-50$ \\
\hline $\begin{array}{l}\text { Age (years) } \\
\quad(<50 \text { years }) \\
\text { Hypertension } \\
\text { Renal disease } \\
\text { Neuropathy }\end{array}$ & $\begin{array}{l}21(31.8) \\
28(42.4) \\
10(15.2) \\
26(39.4)\end{array}$ & 57.3 & $26-78$ \\
\hline $\begin{array}{l}\text { Laser burns } \\
\text { Previous total no. } \\
\text { Index total no. } \\
\text { Cumulative total no. } \\
\text { Spot size }(\mu \mathrm{m}) \\
\text { Power }(\mathrm{W}, \text { green })\end{array}$ & & $\begin{array}{r}2506.8 \\
1754.2 \\
4297.2 \\
403.0 \\
2.05\end{array}$ & $\begin{array}{c}0-9433 \\
461-3302 \\
1485-11164 \\
200-500 \\
0.86-2.6\end{array}$ \\
\hline
\end{tabular}


Table II. Logistic regression using backward stepwise analysis of risk factors predicting outcome of proliferative diabetic retinopathy following argon laser PRP

\begin{tabular}{lcc}
\hline Significant risk factor & Log likelihood ratio significance $(p<0.05)$ & Odds ratio Exp (B) \\
\hline All patients & & 1.0006 \\
Cumulative laser total & 0.0002 & 0.0344 \\
Renal disease & 0.0004 & 0.2460 \\
Age $<50$ years & 0.0265 & - \\
Patients with <6000 laser burns & & 0.0001 \\
Cumulative laser total & $\mathrm{NS}$ & 0.0436 \\
Renal disease & 0.0009 & 0.0111 \\
Age $<50$ years & & \\
\hline
\end{tabular}

\section{RESULTS}

Sixty-six consecutively treated eyes with PDR from 60 patients were studied. All patients received argon laser PRP at the index treatment session with the following mean parameters: $403.0 \mu \mathrm{m}$ spot size, 0.1 second duration and $2.05 \mathrm{~W}$ power setting. The mean number of total argon burns given in the index session was 1754. The mean total number of argon laser burns (calculated from the sum of all previous sessions in patients with a history of treated PDR) was 2506.

Table I shows the distribution of patient characteristics. Sixty-five point two per cent of patients were male and $50 \%$ of patients had insulin-dependent diabetes (IDDM). The mean duration of diabetic disease was 13.3 years, with $53 \%$ patients having had diabetes for over 10 years. Thirty-one point eight per cent patients were over 50 years of age, the mean age being 57.3 years. Forty-two point four per cent patients were hypertensive, $39.4 \%$ had symptomatic diabetic neuropathy and $15.2 \%$ severe renal disease.

Analysis of all patients showed that $56.1 \%$ had evidence of disc new vessel regression at 6 weeks after treatment. The mean cumulative laser total in eyes with regression was 5026 compared with 3367 in non-regressed eyes. Using logistic regression, this difference in the cumulative laser total was highly significant $(p=0.0002)$, as seen in Table II.

Comparison of all patients receiving laser treatment is shown in Fig. 1. Analysis of possible risk factors using logistic regression (Table II), comparing regressed with non-regressed groups, reveals a statistically significant difference $(p<0.05)$ between the two groups with respect to the cumulative number of laser burns $(p=0.0002)$, renal disease $(p=0.0004)$ and age less than 50 years $(p=0.0265)$. The odds ratio for the cumulative laser total was 1.0006 , corresponding to an increase in the likelihood of regression of $0.06 \%$ per argon laser burn applied. In the case of renal disease the odds ratio was 0.034 .

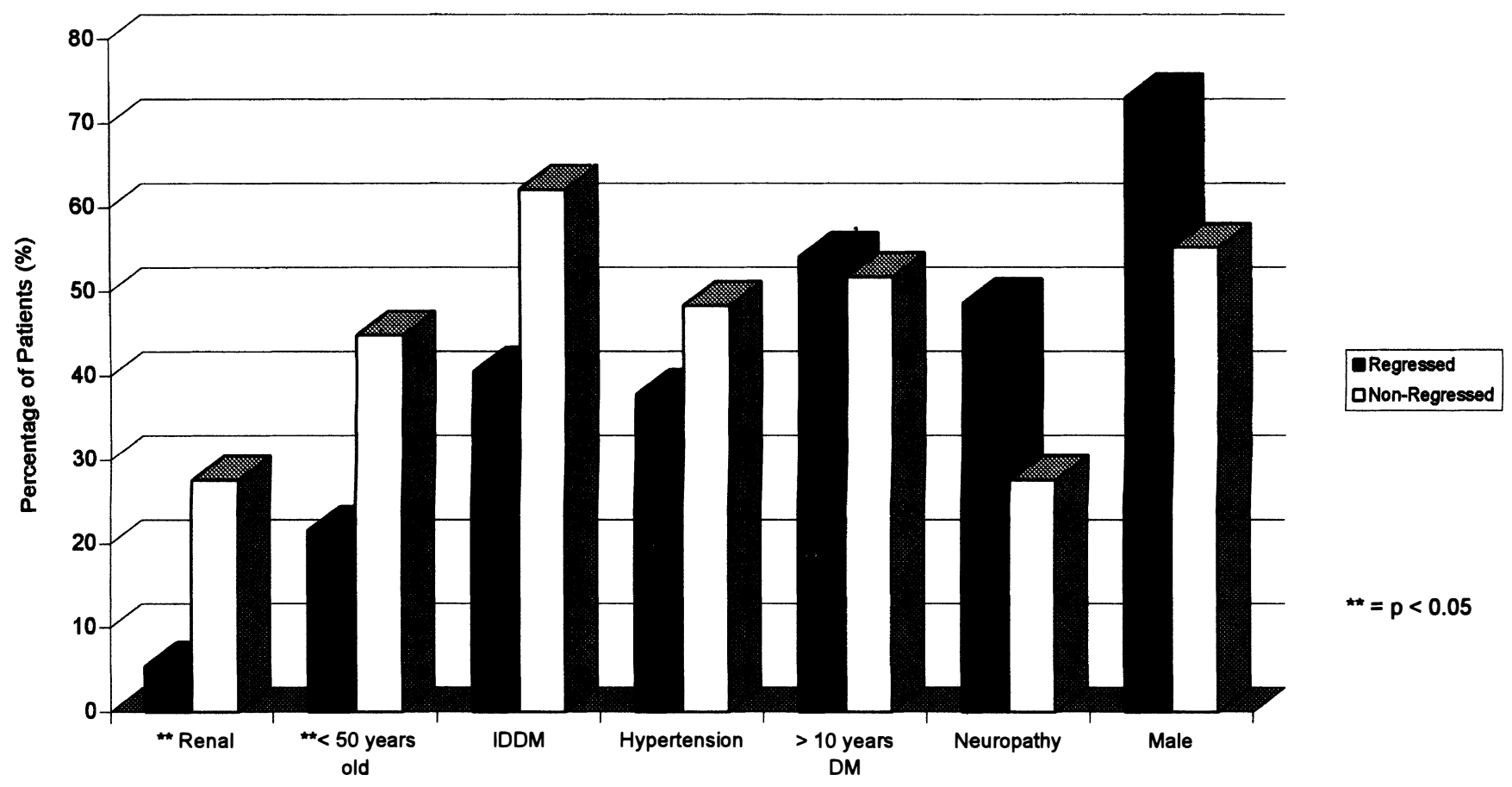

Fig. 1. Comparison of risk factors between the two groups of all patients showing either regression or non-regression following treatment with PRP. Renal disease and age $<50$ years were identified as significant risk factors for non-regression $(* * p<0.05)$. 


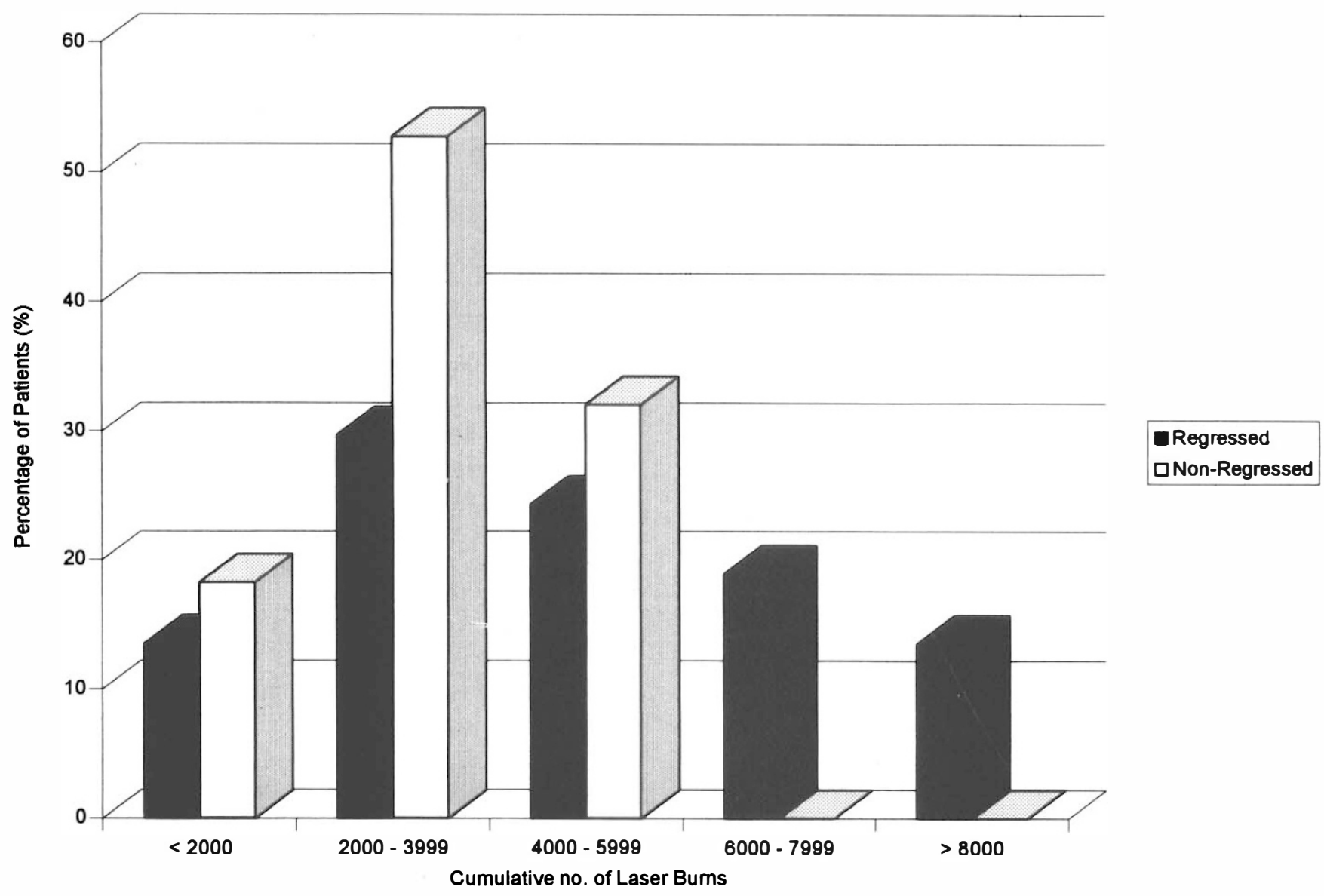

Fig. 2. Distribution of cumulative argon laser burns in regressed and non-regressed patient groups. Mean no. burns: regressed group, 5800; non-regressed group, 3510. Note that all patients showing non-regression received fewer than 6000 laser burns.

In other words, there was an almost 29-fold reduction in the likelihood of regression of proliferative disease in patients with evidence of severe renal disease. The odds ratio for young patients aged 50 years or less was 0.24 . This corresponded to a 4-fold reduction in the likelihood of regression.

Fig. 2 shows the distribution of the total number of laser burns received by patients in either the regressed or non-regressed groups. All patients in the non-regressed group received fewer than 6000 laser burns. There is a substantial overlap between the two groups in the number of patients receiving fewer than 6000 burns. The higher mean in the regressed group is explained by the skew distribution comprising individuals with more than 6000 burns. Comparison of patients in both the regressed and non-regressed groups receiving the same range of treatment (i.e. 0-6000 burns) is shown in Fig. 3 and Table II, using logistic regression. Renal disease is the greatest risk factor within this range of laser treatment $(p=0.0009)$, producing a $10^{4}$-fold decrease in the likelihood of resolution after laser treatment, and young age ( $<50$ years) is the second most important risk factor $(p=0.0111)$, reducing the likelihood of regression by 23 times.

\section{DISCUSSION AND CONCLUSIONS}

We have shown that the outcome of PRP in this study was significantly related $(p=0.0002)$ to the total amount of laser treatment given. The mean number of total laser burns in eyes showing regression at 6 weeks was 5026, whereas in non-regressed eyes it was 3367. Interestingly, all patients with nonregression received fewer than 6000 burns.

The DRS and ETDRS studies demonstrated laser photocoagulation to be very effective therapy for high risk factors of $\mathrm{PDR}^{4,5}$ and established its role in the management of proliferative disease. The DRS was a prospective, randomised, multicentre study of 1758 diabetic patients, evaluating photocoagulation treatment of PDR. One eye of each patient was treated with either argon (800-1600 burns of $500 \mu \mathrm{m}$ spot size or $500-1000$ burns of $1000 \mu \mathrm{m}$ spot size, 0.1 seconds at one sitting) or xenon photocoagulation. After 6 years of follow-up, the untreated eyes had a $34.2 \%$ cumulative rate of severe visual loss $(\mathrm{VA}<5 / 200)$, whereas the cumulative rate in the argon-treated eyes was $17.5 \%$. A $50 \%$ treatment effect was also evident in the xenon-treated group. A similar treatment effect was later observed in the 


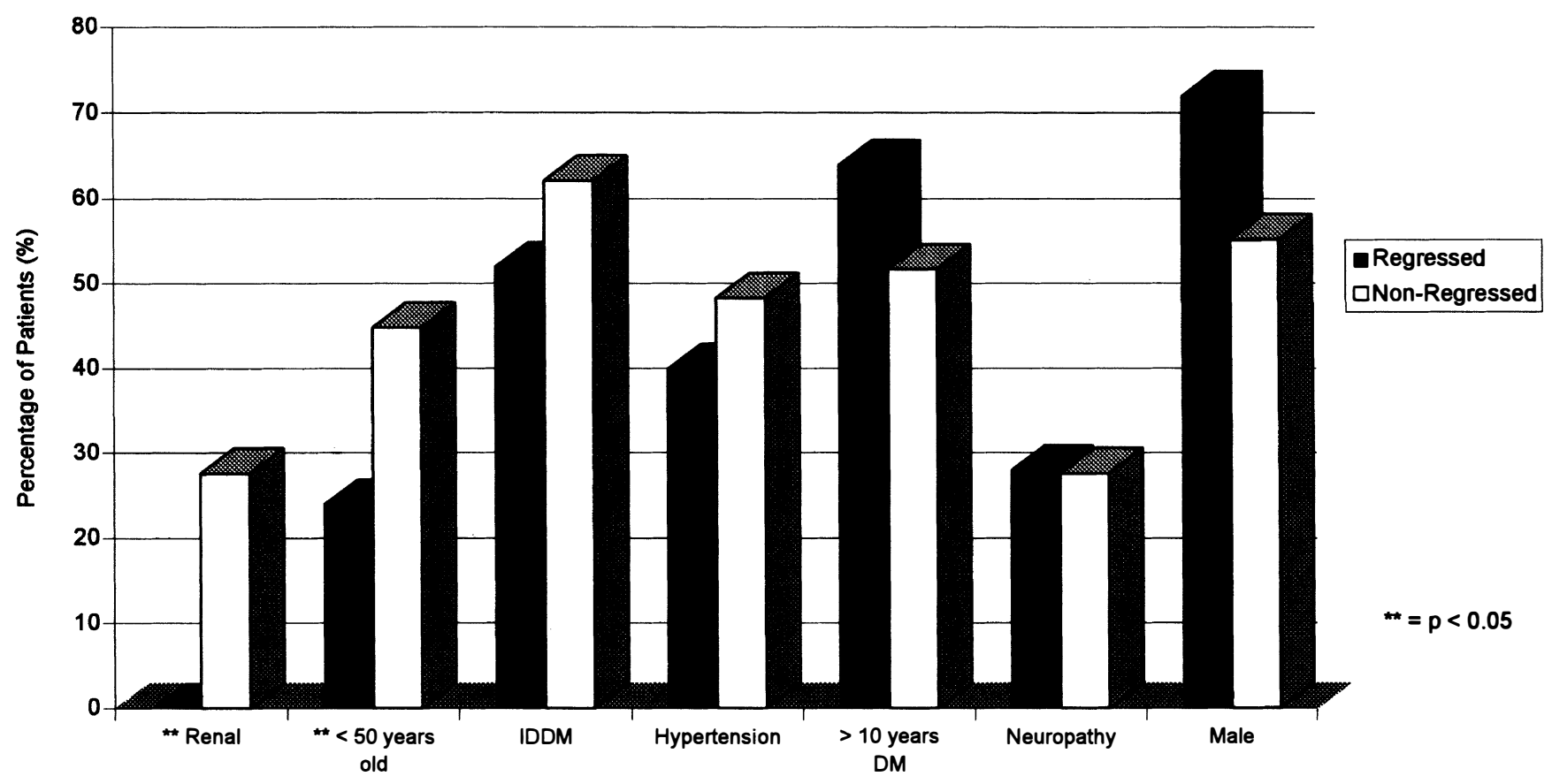

Fig. 3. Comparison of risk factors between regressed and non-regressed groups in patients receiving fewer than 6000 laser burns. Again renal disease and age $<50$ years were identified as significant risḱ factors for non-regression $\left({ }^{* *} p<0.05\right)$.

ETDRS. $^{6,7}$ In this study, which looked at several treatment protocols for PDR and diabetic maculopathy, it was convincingly shown that strategies that included immediate, 'full' PRP (like DRS, argon laser at $500 \mu \mathrm{m}$ spot size, 0.1 second duration and 1200-1600 burns at one sitting) reduced the rate of developing high-risk proliferative retinopathy by approximately $50 \%$, in patients with either early proliferative or severe, non-proliferative disease. This compared with a $25 \%$ reduction in patients treated with immediate but 'mild' (500 $\mu \mathrm{m}$, 0.1 seconds and 400-650 burns at one sitting) photocoagulation. Our 6 week follow-up visit after treatment was identical to the protocol used in the ETDRS and DRS studies. Like these studies, we too have used 6 weeks as a cut-off to assess the initial response to treatment, and we believe that it is current clinical practice to do so, so as to prevent the occurrence of major blinding complications from non-regressed new vessel disease.

The findings of the DRS and ETDRS suggested that the number of burns applied during the 'full' laser PRP protocol was sufficient to induce regression of PDR. However, other studies have shown that the DRS treatment regimen is not effective for all cases of PDR. Aylward et al. ${ }^{8}$ identified a group of diabetic patients requiring extensive PRP. The mean number of burns applied to induce regression was 7225 (range 5136-11 513). Vine ${ }^{9}$ reported on patients not responding to initial PRP of approximately 3000 burns. He found that augmentation laser treatment with an average of 7550 burns achieved a satisfactory response, defined as two or fewer retinopathy risk factors, in $50 \%$ of cases. Another report of successful augmentation lasering for PDR that failed to respond to initial PRP (700-1000 burns, $500 \mu \mathrm{m}, 0.1$ seconds) was shown to achieve a faster regression of retinopathy risk factors than no augmentation, ${ }^{10}$ though precise details of the quantity of additional laser treatment used were not given. None of these studies identified pre-existing systemic conditions that might predict outcome. A very recent study quantitating the amount of retinal ablation required for regression of PDR demonstrated a group of patients with refractory neovascularisation who responded to only extensive treatment $(6500,500 \mu \mathrm{m}$ burns $) .{ }^{11}$ It also showed that the majority of patients with PDR require considerably more treatment than suggested by the DRS. The study identified two risk factors for severe disease: firstly, young age of onset of diabetes (<30 years) and, secondly, duration of diabetes ( $>15$ years).

In our study, analysis of outcome in relation to the presence of diabetic microvascular complications identified renal disease and age ( $<50$ years) as risk factors for non-regression $(p<0.05)$. The duration of disease and insulin dependency in themselves were not identified as risk factors, and neither was the presence of diabetic neuropathy or hypertension. However, we are aware that our analysis might have been different if we had used more stringent definitions for microvascular disease (e.g. as used in the Diabetic Control and Complications Trial ${ }^{12}$ ). Previous studies such as the ETDRS ${ }^{6,7}$ recorded baseline clinical characteristics such as type and duration of diabetes, age, sex, hypertension, $\mathrm{HbA}_{1 \mathrm{c}}$ 
and serum creatinine, but no analysis of these factors in relation to response to treatment was ever performed. This was also the case in studies involving augmentation PRP. ${ }^{8,10}$

A 6 year study of diabetic Pima Indians showed the incidence of haemorrhagic and exudative retinal lesions was strongly related to duration of disease and insulin dependency. ${ }^{13}$ This same study identified hypertension as a risk factor for exudative diabetic retinopathy, with a clear correlation existing between an elevated systolic blood pressure and the presence of exudative lesions. However, the effect of these factors on PDR has not been studied. ${ }^{1}$

The Diabetes Control and Complications Trial (DCCT) assessed diabetic complications in relation to the intensity of treatment. ${ }^{12}$ Diabetic retinopathy was shown to be greatly affected by the degree of control of the disease. Intensive therapy retarded by $47 \%$ the development of proliferative or severe nonproliferative retinopathy in patients with established retinopathy. Nephropathy (as measured by albuminuria and microalbuminurea) and neuropathy (defined as an abnormal neurological examination that was consistent with the presence of peripheral sensorimotor neuropathy plus either abnormal nerve conduction in at least two peripheral nerves or unequivocally abnormal autonomic nerve-testing) in patients with IDDM were also affected by disease control. These findings support a correlation between microvascularity and diabetic control, and suggest that diabetic retinopathy, nephropathy and neuropathy may be used as markers of diabetic disease control. Hence, the more poorly controlled the patient, the greater the incidence of microvascular complications manifested, for example, by PDR. If glycaemic control is good, then the incidence of diabetic nephropathy is reduced. The progression of PDR has been shown to be affected by the status of diabetic renal disease, as shown in patients receiving a combined renal and pancreatic transplant ${ }^{14}$ who demonstrated a reduction in the severity of PDR on recovery of renal function.

Our findings are in agreement with other authors,${ }^{11}$ and show that the majority of patients with established disc neovascularisation (NVDs) require much more treatment than that recommended in the DRS. We are aware that such heavy amounts of laser burns will lead to significant visual dysfunction (restricted visual field, night blindness, etc.), but feel it is more important to recognise the need for adequate laser treatment to control neovascularisation and so prevent the potentially devastating complication of blindness. We suggest that patients with renal disease represent a high-risk group of diabetics with PDR with evidence of severe disease. These patients should be identified, monitored more closely and treated more aggressively from the start, as our results suggest that they require more extensive PRP.

Key words: Panretinal photocoagulation, Proliferative diabetic retinopathy, Diabetic vascular disease.

\section{REFERENCES}

1. Ulbig M. Hamilton A. Factors influencing the natural history of diabetic retinopathy. Eye 1993;7:242-9.

2. Office of Population Census and Surveys. Causes of blindness and partial sight in England and Wales 1990-1991. Studies on medical and population subjects no. 57. London: HMSO, 1995:13570th edn.

3. Beetham W. Visual prognosis of proliferating diabetic retinopathy. Br J Ophthalmol 1963;47:611-9.

4. Diabetic Retinopathy Study Research Group. Photocoagulation treatment of proliferative diabetic retinopathy: clinical application of diabetic retinopathy study (DRS) findings. DRS report no. 8. Ophthalmology 1981;88:583-600.

5. Diabetic Retinopathy Study Research Group. Four risk factors for severe visual loss in diabetic retinopathy: the third report from the diabetic retinopathy study. Arch Ophthalmol 1979;97:654-5.

6. Early Treatment Diabetic Retinopathy Study Research Group. Early treatment diabetic retinopathy study design and baseline patient characteristics. ETDRS report no. 7. Ophthalmology 1991;98:741-56.

7. Early Treatment Diabetic Retinopathy Study Research Group. Early photocoagulation for diabetic retinopathy. ETDRS report no. 9. Ophthalmology 1991;98:766-85.

8. Aylward GW, Pearson RV, Jagger JD, Hamilton AM. Extensive argon laser photocoagulation in the treatment of proliferative diabetic retinopathy. $\mathrm{Br} \mathrm{J}$ Ophthalmol 1989;73:197-201.

9. Vine AK. The efficacy of additional argon laser photocoagulation for persistent, severe proliferative diabetic retinopathy. Ophthalmology 1985;92:1532-7.

10. Doft B, Metz D, Kelsey S. Augmentation laser for proliferative diabetic retinopathy that fails to respond to initial panretinal photocoagulation. Ophthalmology 1992;99:1728-35.

11. Reddy VM, Zamora RL, Olk RJ. Quantitation of retinal ablation in proliferative diabetic retinopathy. Am J Ophthalmol 1995;119:760-6.

12. The Diabetes Control and Complications Research Group. The effect of intensive treatment of diabetes on the development and progression of longterm complications in insulin-dependent diabetes mellitus. N Engl J Med 1993;329:977-86.

13. Knowler W, Bennett P, Ballintine E. Increased incidence of retinopathy in diabetics with elevated blood pressure: a six-year follow-up study of Pima Indians. N Engl J Med 1980;302:645-50.

14. Ulbig M, Kampik A, Thurau S, Land W. Long-term follow-up of diabetic retinopathy for up to 71 months after combined renal and pancreatic transplantation. Graefes Arch Clin Exp Ophthalmol 1991;229:242-5. 